\title{
The Use of Geotechnical Methods to Determine the Strength Parameters of the Substrate in Terms of Operation and Safety of Mortar Use
}

\author{
Grzegorz Bartnik ${ }^{1}$, Kazimierz Józefiak ${ }^{1}$, Małgorzata Superczyńska ${ }^{1}$, Magdalena Czerwińska ${ }^{2}$, Witold Krajewski ${ }^{2}$, \\ Józef Legieć ${ }^{2}$, Tadeusz Kuśnierz ${ }^{2}$ and Mariusz Magier ${ }^{3, *}$
}

1 Warsaw University of Technology, Institute of Roads and Bridges, Faculty of Civil Engineering, 16 Armii Ludowej Ave., 00-637, Poland; G.Bartnik@il.pw.edu.pl (G.B.); k.jozefiak@il.pw.edu.pl (K.J); M.Superczynska@il.pw.edu.pl (M.S.)

2 Military Institute of Armament Technology, Prym. S. Wyszyńskiego 7 Str., 05-220 Zielonka, Poland; czerwinskam@witu.mil.pl (M.C); krajewskiw@witu.mil.pl (W.K.); legiecj@witu.mil.pl (J.L.); kusnierzt@witu.mil.pl (T.K.)

3 Warsaw University of Technology, Institute of Mechanics and Printing, Faculty of Mechanical and Industrial Engineering, Narbutta 85 Str., 02-524 Warsaw, Poland

* Correspondence: mariusz.magier@pw.edu.pl (M.M.)

\begin{abstract}
During firing from a mortar, an important issue is the parameters of compressibility of the ground on which the mortar is placed. This affects the operation of the mortar (including safety). During the qualification tests of the mortar, the influence of different types of substrate on its strength and work during shooting should be examined. Until now in the Polish standardization documents was no clear description about the substrate parameters used for this kind of tests. Analysis of the literature also did not allow to determine the dependence of the mortars displacement in the function of the type of substrate and its geotechnical parameters. Therefore, the authors carried out analytical and experimental analysis of the use of geotechnical methods to determine the parameters of the substrate for mortar testing. The paper presents the proposed types of standardized soil and their research methods. Preliminary tests were also carried out using the prescribed measurement methods and a comparative mortar firing test. Subsequently, an analysis of the results was carried out and the possibilities of using the proposed methods of measuring the parameters of soil compressibility were determined.
\end{abstract}

Keywords: mortar, strength properties, operational safety, organic soils, weak soils, deformation modulus, dynamic deformations

\section{Introduction}

The main source of artillery support for combat troops in direct contact with the enemy are towed mortars. Compared with other types of artillery equipment, they are characterized by the following characteristics:

- Shooting is carried out at the angles of the sight $\mathrm{c} \geq 45^{\circ}$;

- The recoil force created during the shot is transmitted via the base plate directly to the ground. The towed mortar does not have any devices that would absorb (decrease) the impact of the recoil force pulse on the base plate;

- In the majority, mortars have smoothbore tube (barrel). For this reason the projectiles fired from mortar are fin-stabilized [1].

A mortar with a typical design for this type of weapon is a $98 \mathrm{~mm}$ M98 (Figure 1) mortar manufactured by Huta Stalowa Wola S.A-Poland [2]. 


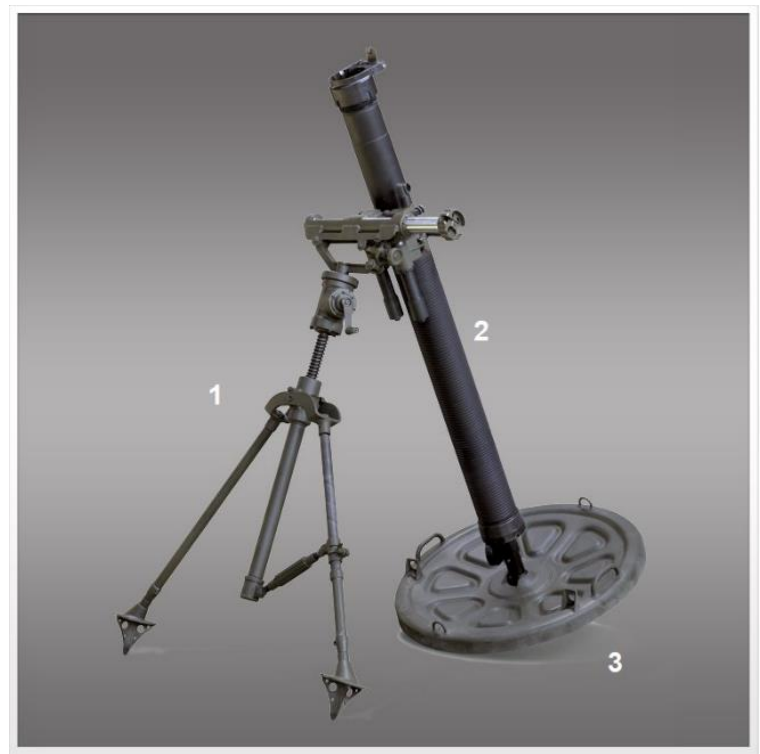

Figure 1. Mortar M98 (HSW S.A.), 1 - bipod unit with elevation and direction mechanisms, 2 - mortar tube, 3 - base plate.

The tube is used to give the required velocity and flight's direction the projectile . For the correct positioning the tube, the bipod assembly is used,. The base plate is used to support the mortar during the shot and transmit the recoil impulse to the ground.

The issues of cooperation of the mortar base plate with the ground are not widely described in world literature. The main emphasis in research is on the analysis of the stress and strength of the mortar itself (including the base plate). The paper [3] discuss the studies of effort in various elements of the mortar during shooting from gravel ground. Tests of this type allow to determine the safety of use (strength) of the mortar. The paper [4] presents a shotless low-cost method of analyzing the strength of a base plate. Such research is the basis for optimizing the design of the mortar. The paper [5] show an example of optimizing the construction of a mortar's base plate in terms of reducing its weight while maintaining the required strength and rigidity. The weight of the mortar is very important parameter, because it is supposed to be a light and mobile weapon. Studies on mortar recoil have been presented for pyrotechnic mortars at work [6], but they show the results of the much lower loads than military mortars. For the issues of the relationships between the mortar and the substrate with different parameters, the authors did not find any studies.

\section{Dynamics of mortars movement during the firing process}

In order to give the mortar projectile an initial velocity providing the required distance, appropriate propelling charges shall be used. These charges during the shot burn create a high pressure of gunpowder gases that affect both the projectile and the bottom of the barrel. Although the mass of the propelling charge is variable (depending on the required distance of the projectile), in this article the work of the mortar on the full propelling charge will be described, ensuring both the highest velocity of the projectile and the highest pressure of gunpowder gases inside the tube [7-9]. Figures 2 and 3 show the pressure $p(t)$ diagrams inside the $60 \mathrm{~mm}$ and $98 \mathrm{~mm}$ mortar barrel tube during firing. 


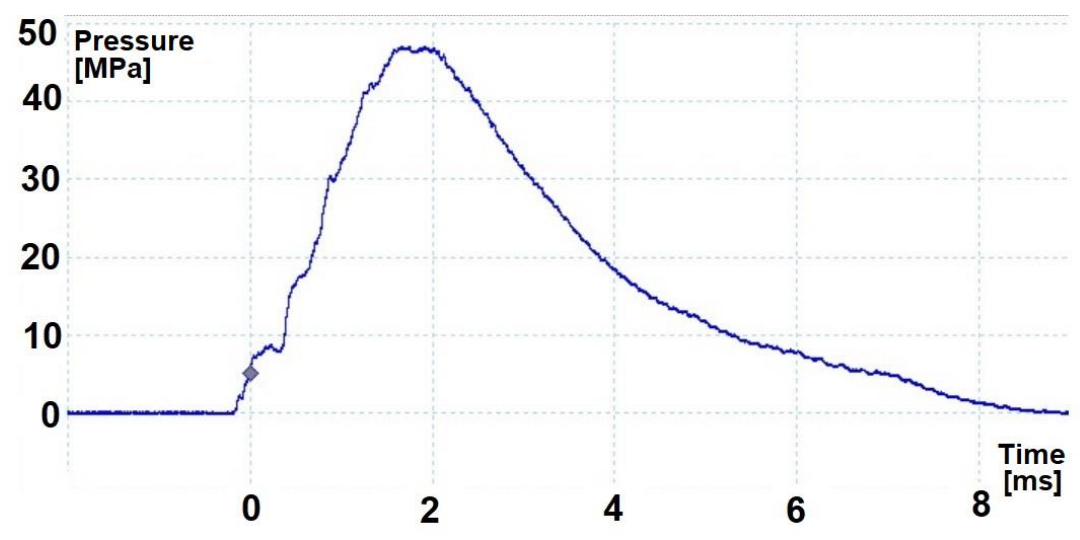

Figure 2. Example of pressure $p(t)$ in the tube of a $60 \mathrm{~mm}$ mortar during firing.

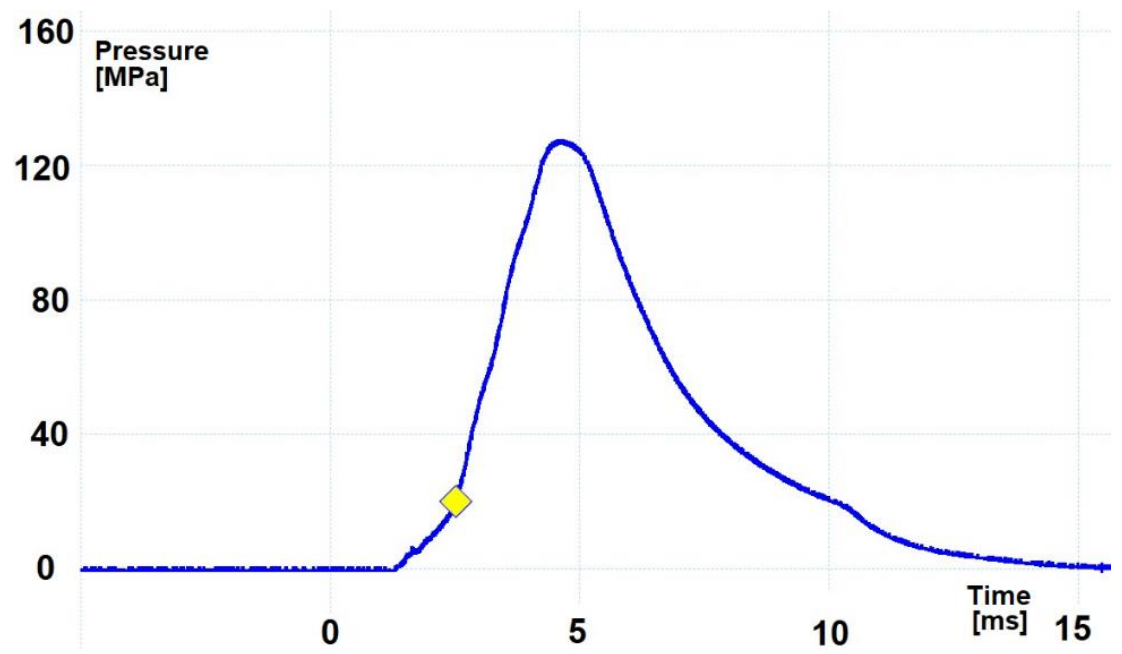

Figure 3. Example of pressure $p(t)$ in the tube of a $98 \mathrm{~mm}$ mortar during firing.

In the case of a $60 \mathrm{~mm}$ mortar, the maximum pressure shown in Figure 2 is $47,5 \mathrm{MPa}$ which, after taking into account the surface of the bottom of the barrel of $\approx 0,002827 \mathrm{~m}^{2}$, generates a maximum force acting on the mortar $\approx 134,3 \mathrm{kN}$. For a $98 \mathrm{~mm}$ mortar, these are $127 \mathrm{MPa}, \approx 0,007543 \mathrm{~m}^{2}$ and $\approx 958 \mathrm{kN}$, respectively. Because the pressure in the tube and the associated forces acting on the mortar are quickly changing, most often the total impulse of momentum force transmitted to the gun during the shot is used to describe the recoil phenomenon. Table 1 presents various parameters related to the phenomenon of recoil in mortars operated by the Polish Armed Forces when firing on full charges. An impulse of the recoil force is understood as the momentum transmitted to the mortar elements participating in the recoil. The theoretical recoil velocity is the value that the mortar elements reached during recoil if they had not been affected by braking forces.

Table 1. The recoil parameters in mortars operated by the Polish Armed Forces.

\begin{tabular}{cccccc}
\hline L. p. & $\begin{array}{c}\text { Caliber of } \\
\text { mortar }\end{array}$ & $\begin{array}{c}\text { Recoil force } \\
\text { pulse [Ns] }\end{array}$ & $\begin{array}{c}\text { Average } \\
\text { duration of the } \\
\text { shot } \\
{[\mathbf{m s}]}\end{array}$ & $\begin{array}{c}\text { Weight of } \\
\text { rejected elements recoil velocity } \\
{[\mathbf{k g}]}\end{array}$ & $\begin{array}{c}\text { Theoretical } \\
\text { [m/s] }\end{array}$ \\
\hline 1 & $60 \mathrm{~mm}$ & 320 & 8 & 18,3 & $\approx 17,5$ \\
\hline 2 & $98 \mathrm{~mm}$ & 3620 & 10 & 120 & $\approx 30,2$ \\
\hline 3 & $120 \mathrm{~mm}$ & 4400 & 14 & 207 & $\approx 21,3$ \\
\hline
\end{tabular}

To brake the barrel of the mortar and ensure its stability during the shot, a base plate is used, which transmits the recoil force to the ground. Due to the much larger diameter of the base plate in relation to the diameter of the barrel, the pressures that the base plate 
can affect the substrate are much smaller. Nevertheless, for a $60 \mathrm{~mm}$ mortar, the maximum pressure that the base plate can affect the substrate is 1,57 MPa. In the case of a $98 \mathrm{~mm}$ mortar, it is $1,9 \mathrm{MPa}$. In most cases the shooting is carried out by the mortar adopted to the deformable substrate, it will delve into ground together with the base plate during the shot. Figure 4 shows a sequence of frames from a movie recorded with a high speed camera during a mortar shot, showing the sinking of the base plate into the sandy soil. The Figure 4 show the frames recorded every $10 \mathrm{~ms}$.
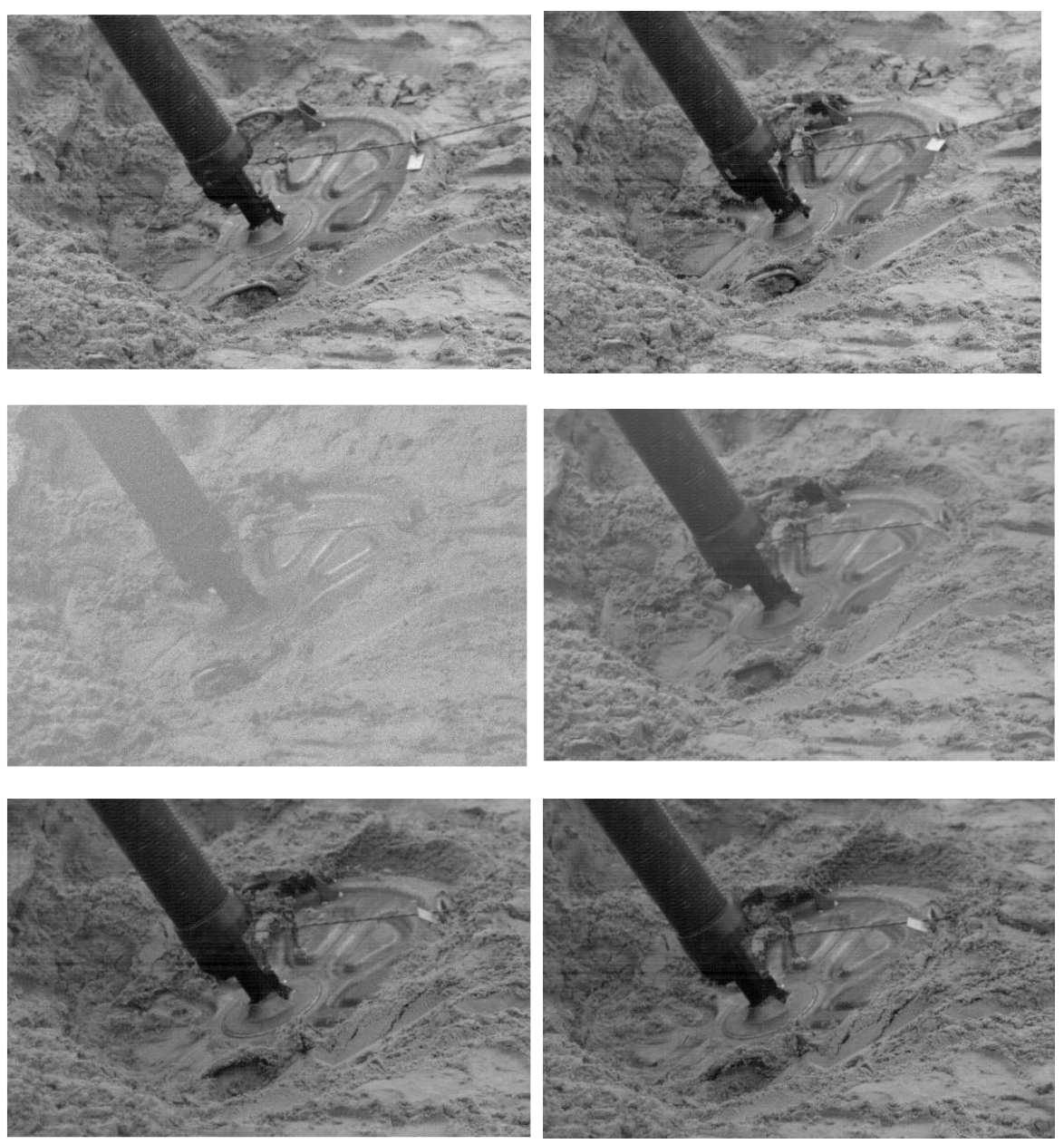

Figure 4. Mortar's recoil process on sandy soil.

For a substrate susceptible to deformation during firing, the reduced force, which is the sum of the recoil force and the reaction force of the substrate to the base plate, create the mortars velocity in the oppositive direction of the projectile velocity, which causes the mortars plunge into the ground. After the projectile leaves the tube and the pressure of the gunpowder gases in the barrel decreases to atmospheric pressure, the force of inertia of the mortar causes its further recess into the ground [10-11]. At this stage, the only significant force acting on the mortar is the reaction force of the ground, which inhibits its recoil speed. After stopping the mortar, as a result of the substrate's response, mortar may move in the opposite direction - called "jumping".

The quality requirements for mortars contain the possibility of firing from them after placing on substrates with a wide range of properties. The cooperation of the mortar with the subgrade is largely dependent on its susceptibility to deformation [12-13]. For nonsusceptible substrate the "jumping" of the mortar and its displacement or sliding of the base plate on the ground may occur. With a substrate very susceptible to deformation, the mortar together with the base plate can sink to a significant (relative to its mortar) depth or also slip. A large displacement of the mortar when delving into susceptible ground or 
during its "jumping" on a non-susceptible and elastic ground significantly prolongs the fulfill of the fire task, because before each subsequent shot it is required to re-set the mortar. A significant influence on the results of mortar shooting is also the stability during the shot (especially during the movement of the projectile inside the gun tube). Even a slight deviation of the mortar barrel (by $1^{\circ}$ ) in the direction will cause a deviation of the point of hit of the projectile (at a firing distance of $2000 \mathrm{~m}$ ) - by over $32 \mathrm{~m}$, and with a shooting distance of $5000 \mathrm{~m}$ - by over $80 \mathrm{~m}$ [14]. The impact of mortar tube's deflection in the vertical plane is several times smaller. Therefore, artillery practice often assumes (in the case of placing the mortar on susceptible ground) giving the first shot in order to place the mortar in the ground, the so-called "settling shot".

Another important aspect resulting from the cooperation of the mortar with the ground is the safety and reliability. Mortar firing on a low-susceptible ground significantly increases the effort of the main mortars parts. This applies in particular to the base plate and the connection between the base plate and the barrel. Therefore, the development of a base plate durable to multiple impulse loads, and the same time ensuring stable behavior of a mortar placed on various types of substrates (soils) requires determining the relationship between the base plate and the ground during the shot. When firing from a very susceptible ground, a large displacement of the mortar may lead to damage to the joining of the barrel support, and in extreme cases due to the slipping of the base plate to overturn the mortar. For the mortars of larger calibers even more than $200 \mathrm{~kg}$ weight, this may pose a threat to the safety of the crew.

\section{The issue of interaction between mortar and substrate from the standardization point of view}

The issue of interaction between mortar and substrate significantly affects the problem of safety and reliability of the mortar use, is regulated mainly by the Defense Standards. It is a series of documents supervised by the Polish Military Center for Quality Standardization and Codification for the normalization of issues related to weapons equipment. The standard NO-10-A216:2012 [15] contains a set of requirements and tests that must be fulfill by mortars introduced into the Armed Forces.

Because of operational safety is an overriding requirement for armament equipment, the standard NO-10-A216:2012 [15] clearly requires the safety and correct use of mortars placed on various types of ground. The mortar test procedure contained the requires conducting research shooting on "soft" and "hard" soils. The following type of soil are determined:

1. hard soils (calcareous, stony, compacted clay and frozen soils),

2. medium soils (sandy, clayey and turf soils),

3. soft soils (muddy, "arable" soils, peat and "plump sand").

Preliminary analysis of the issue showed that the greatest complication of this issue occurs in the case of soft soils. Apart from the inconsistency in naming, the above-mentioned names of soft soil types do not appear in the geotechnical standards [16, 17]. The soft soils in the engineering practice should be treated as low, insufficient bearing capacity substrate. "Weak soil" may be defined as a soil layer that do not fulfill the requirements that result from conditions of compressibility, stability or suitability for use for a particular object or element of structure [18]. In engineering practice, the parameterization of weak soils is practically limited to determining their depth of deposition in the active zone of the substrate, usually they are not described by the values of geotechnical parameters. They are reinforced in a way that depends on the depth of deposition, the type of soil and designed construction. Another aspect that makes geotechnical investigation of the substrate difficult are the limitations resulting from the technical capabilities of the research equipment, which is not adapted to determine the parameters of weak soils. Moreover there are no appropriate standards to interpret the obtained results of this type of soil tests. In addition, when choosing a test method, the conditions of the proving ground should be taken into account as a place where test shooting will take place and where it is 
necessary to prepare and examine the ground for the shooting position. Therefore, the ground should:

- $\quad$ be formed without specialist geotechnical (geological) knowledge and heavy equipment,

- $\quad$ provide repeatability of obtained ground conditions,

- be identified by a parameter which does not require the use of complicated or timeconsuming methods and can be determined in field conditions,

- allow for short substrate preparation,

- be easily obtainable.

\section{Soft substrate}

\subsection{Selection of a model substrate}

The standard [15] introduces the following types of soft soils: muddy, arable soils, peat and plump sand. Taking into account the potential difficulties with the use of cohesive soils as a weak layer during their preparation, formation and compaction resulting from the need to moisten them (to achieve at least plastic state), the modeling of "muddy soil" or "arable soil" was omitted. It was decided to use "peaty soil" (resigning from modeling "plump sand" due to the possibility of obtaining a much weaker soil and a wider range of "weaknesses" of this substrate). As a peat substrate was used available garden peat.

Initially, it was assumed that the substrate model would be characterized by parameters describing deformability. The bearing capacity of soils increases with compaction, then it is necessary to control the amount of peat to obtain the required values of deformation parameters. However, in engineering practice for peats there is no test procedure to determine the values of the state of compaction parameters, i.e., relative density (density index) ID or relative compaction (degree of compaction) Is.

The density index $I_{D}$ determines the condition of the cohesionless soils which peat is not. In accordance with the standard BN-77/8931-12:1977 [19], there is no test procedure to determine the value of the maximum dry density of the peat $\rho \mathrm{ds}$ which is taken into account in the calculation of Is. Determining the value of the $\rho_{\mathrm{d}}$ for peat is quite difficult due to the need to take a sample with an undisturbed structure. It is also not possible to use indirect methods for determining compaction parameters such as dynamic probing.

In the authors opinion, it is possible to determine the bulk density of the substrate model and control this parameter in the conditions of substrate formation process. It should be noted that the value of the density depends on the moisture, which in the case of peat can be within a wide range. The parameter that better describes the state of compaction is the dry density, however this method requires the determination of the moisture value, which in practice in field conditions is not feasible.

\subsection{Research methods}

The methods of assessing the deformability in situ conditions include: the method of static plate load test and dynamic load plate test. These methods allow for direct measurement of the soil response to an applied load described by the following parameters: the deformation modulus $E$ and the dynamic modulus of deformation $E v d$.

The static plate load test is commonly used in road construction in the form of loads generated by the VSS apparatus (In-Germany: Verein Schweizer Strassenfachmänner). The deformation modulus in the VSS test is defined as the ratio of the unit load increment to the deformation increment of the tested layer in a fixed range of unit loads [20]. Double static loading of the test surface allows to calculate the primary $E_{1}$ and secondary $E_{2}$ deformation modules. In the case of assessment of the stiffness of the thick ground layers, large-scale load tests are used [21].

The dynamic load plate test by lightweight deflectometer was developed initially in Germany [22, 23], as a device for in-situ testing of stiffness and compaction of the substrate and road construction layers, as well as an alternative to static testing. The main 
advantage of this method is mobility and short test time. In the dynamic load plate test, the amplitude of plate displacement caused by a single impact pulse is measured.

\subsection{Test stand}

To assess the possibility of using peat as a model substrate of the shooting station, a preliminary research program was planned and carried out. The program includes verification of the use of peat in the test stand and the validity of the selected research methods. For this purpose, a test stand was made in the form of a wooden box with a volume of 0,7 $\mathrm{m}^{3}$ in which previously weighted peat was placed. About 197,4 kg of peat was placed in the box, which allows to determine the bulk density at $282 \mathrm{~kg} / \mathrm{m}^{3}$.

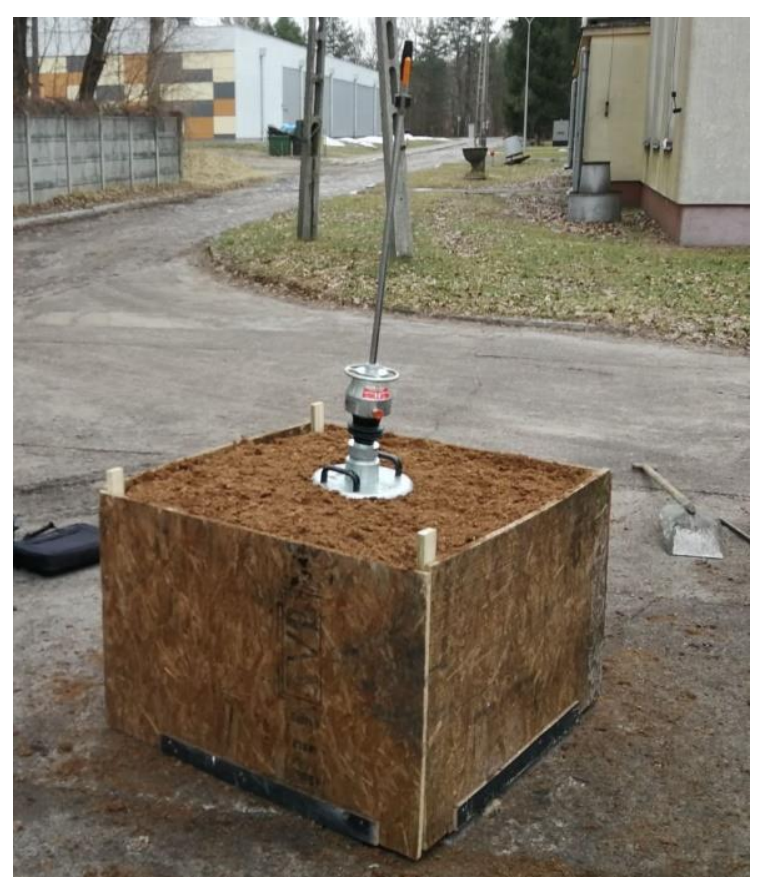

Figure 5. Test stand.

\subsection{Static load method}

The static plate load test were carried out using a standard rigid plate (diameter 30 $\mathrm{cm}$ ) included in the VSS apparatus. In practice, due to the lack of space, the use of dial sensors and the rack attached to the apparatus for settling measurements has been omitted.

The load $\sim 215 \mathrm{~kg}$ consisted of a stack of 7 steel plates $(20 \div 30 \mathrm{~kg}$ each) and VSS plate. Applying the entire load is divided into 7 stages.

The measurement of displacement was carried out using the geodetic method at four points on the surface of the rigid plate. This allowed to eliminate possible stack tilts, and the average value of settlements measured at individual points was taken as a result.

The load consisted of two phases: the primary loading and the subsequent unloading. Each phase was carried out in stages, and the final load value was selected to obtain pressures similar to those generated by humans (approx. $30 \mathrm{kPa}$ ). It was assumed that in field conditions, human-induced subsidence could be used as an initial rating of the ground quality of the shooting position.

\subsection{Dynamic load method}

The dynamic load plate test consists of measuring the deformation of the ground surface, caused by a short-term force impulse. The impulse load is generated by a freely falling mass and transmitted through the pressure plate to the ground. This method is treated as a short-term quasistical load of the plate on the substrate. The free fall of the weight, falling along the guide rod on the spring-damper element, generates an impact- 
like load $P_{D}$ on the load plate. The pulse is transmitted through the load plate to the surface of the soil medium, generating a contact stress $\sigma_{D}=100 \mathrm{kPa}$ and causing the substrate settlement equal to the displacement uD of the rigid load plate [24].

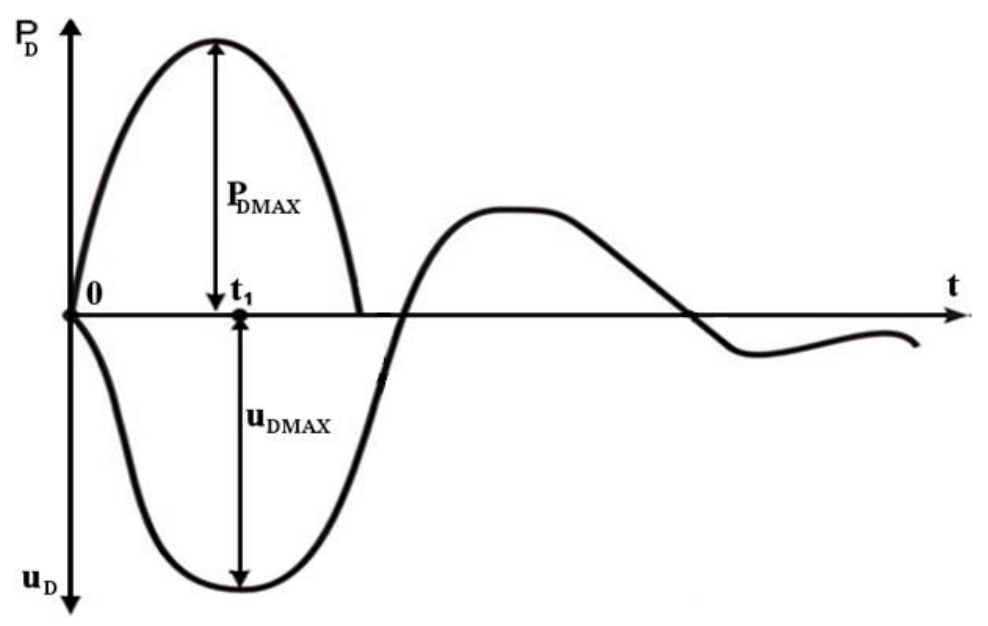

Figure 6. Variability in time $t$ of loading force $P_{D}$ and displacement uD in a dynamic test [20].

In the research schedule, it was assumed that the determination of the dynamic modulus of deformation $E_{v d}$ will be carried out with a lightweight deflectometer ZORN ZFG 2000. During the tests, it turned out that the magnitude of the generated acceleration exceeds the measuring capacity of the accelerometer amounting to $\pm 100 \mathrm{~g}$, which corresponds to a displacement of $0,2 \div 30 \mathrm{~mm} \pm 0,02 \mathrm{~mm}$. Therefore, a Photron high-speed camera was used to determine the course of displacement during the dynamic test. The image was recorded at a frequency of 6000 frames per second, but the analysis of the dynamics of this phenomenon indicates that 2000 frames per second is sufficient. The recordings from the camera were subjected to imaging analysis in order to determine the changes in the position of the measuring points during the test. During one study, three drops were performed. The analysis took into account three reference points ( $00 ; p 1 ; p 2)$ marked on the device used (Figure 7).

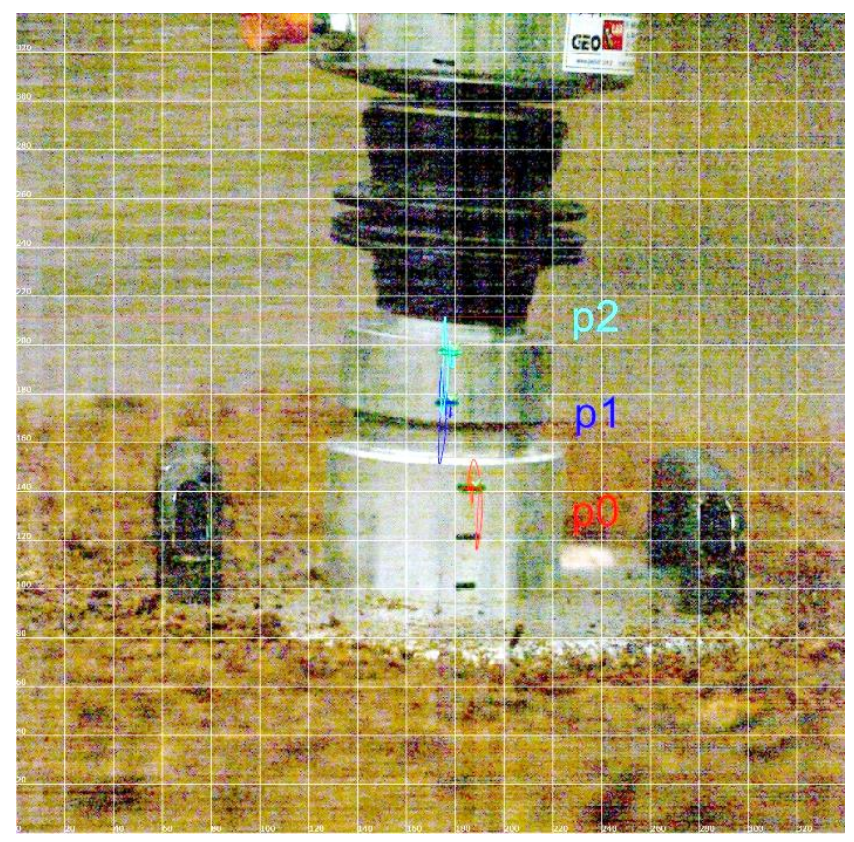

Figure 7. Displacement of reference points during impact.

\subsection{Results}


Starting from the analysis of settlement of the substrate as isotropic and homogeneous elastic half-space, the value of the substrate deformation modulus $E$ from static plate load tests and the dynamic modulus of deformation $E_{v d}$ from dynamic plate load tests can be determined from the formula [25]:

$$
E, E_{v d}=\left(1-v^{2}\right) \omega \frac{D \Delta p}{\Delta s}
$$

Where: $v$ - Poisson's ratio, $\omega$ - the shape factor, which for a circular rigid plate takes the value $\omega=0,79, D$-diameter of the loading plate [m], $\Delta p$-the increase in load [Pa], $\Delta s$ - the increase in settlement for given increase in load [m].

For a dynamic load plate test $\Delta p=0,1 \mathrm{MPa}, \Delta s$ is the maximum displacement of the loading plate.

The results of the static tests calculated using formula (1) are shown in Table 1. Figure 8 presents the dependence the substrate settlement on the used loads. The shape of this graph suggests the linear soil response to the applied loads. In this case, in the calculation of the modules, the value of the Poisson's ratio for peat $v=0,2$ and the plate diameter $D=30 \mathrm{~cm}$ were assumed.

Table 1. Static load test results.

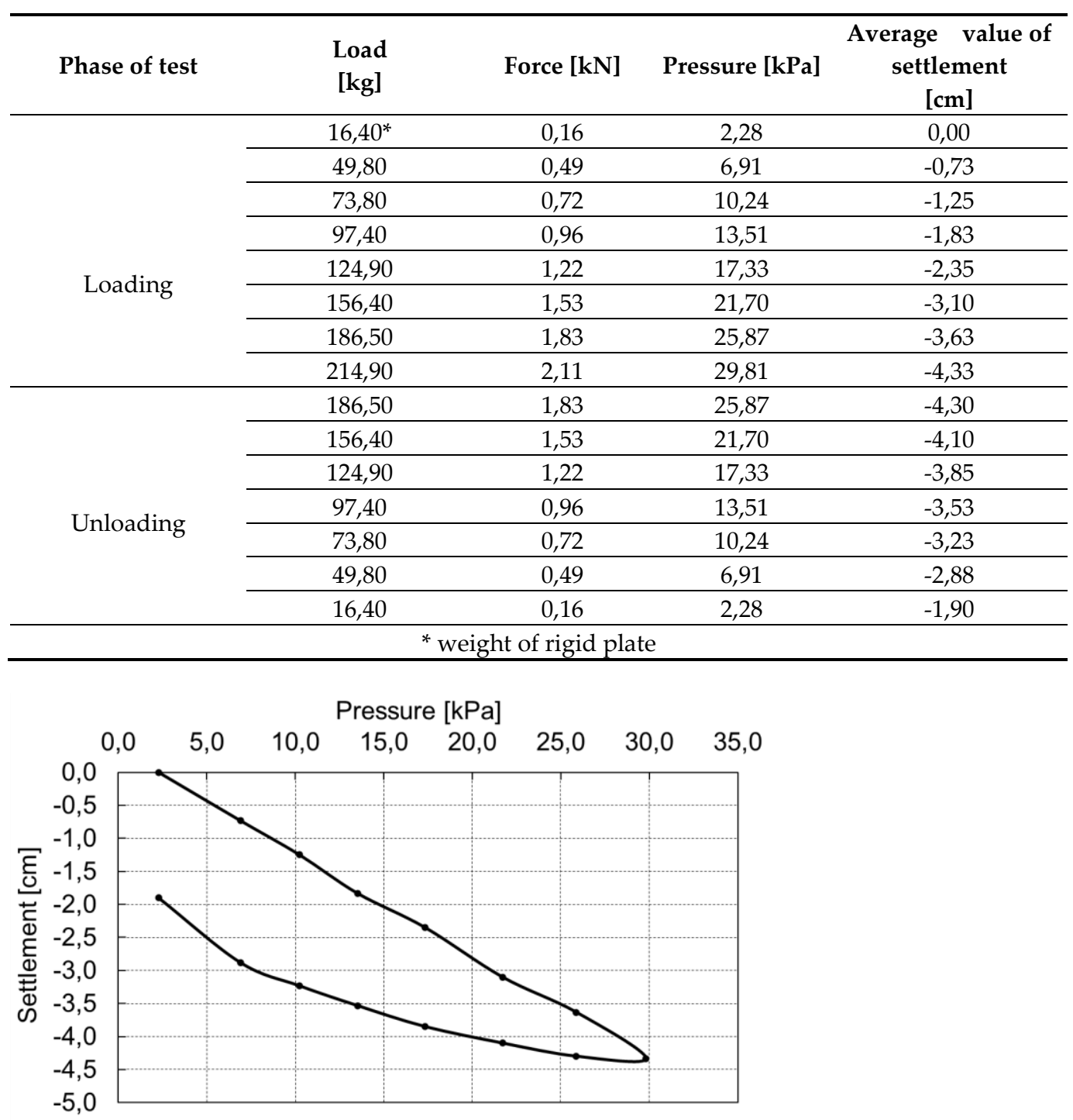

Figure 8. Pressure-settlement relationship for static load test. Table 2.

The values of the primary static deformation modulus for each stage are listed in the 
Table 2. Primary static deformation modules.

\begin{tabular}{|c|c|c|c|c|c|}
\hline $\begin{array}{c}\text { Pressure } p \\
{[\mathrm{kPa}]}\end{array}$ & $\begin{array}{c}\text { Increase in pres- } \\
\text { sure } \\
\Delta \mathrm{p}[\mathrm{kPa}]\end{array}$ & $\begin{array}{l}\text { Settlement } \\
\mathrm{s}[\mathrm{cm}]\end{array}$ & $\begin{array}{c}\text { Increase in } \\
\text { settlement } \Delta \mathrm{s} \\
{[\mathrm{cm}]}\end{array}$ & $\begin{array}{r}\text { Primary } \\
\text { matior } \\
\mathrm{E}_{1}\end{array}$ & $\begin{array}{l}\text { tatic defor- } \\
\text { modulus } \\
{[\mathrm{kPa}]}\end{array}$ \\
\hline 2,28 & 0,00 & $-0,00$ & 0,00 & 0,00 & \multirow{8}{*}{$\begin{array}{c}144,6 \\
\text { (average) }\end{array}$} \\
\hline 6,91 & 4,63 & $-0,73$ & 0,73 & 144,3 & \\
\hline 10,24 & 3,33 & $-1,25$ & 0,52 & 145,7 & \\
\hline 13,51 & 3,27 & $-1,83$ & 0,58 & 128,3 & \\
\hline 17,33 & 3,82 & $-2,35$ & 0,52 & 167,1 & \\
\hline 21,70 & 4,37 & $-3,10$ & 0,75 & 132,6 & \\
\hline 25,87 & 4,17 & $-3,63$ & 0,53 & 179,0 & \\
\hline 29,81 & 3,94 & $-4,33$ & 0,7 & 128,1 & \\
\hline
\end{tabular}

The course of the impact was recorded in the dynamic plate test with the use of a high-speed camera. On this basis, the values of the settlement of the loaded substrate were determined for the entire impact phase. Below there are presented the diagrams (Figure 911) of displacement during three consecutive drops for the p0 point. Positive displacement values on the graphs are related to the detachment of the plate from the tested substrate.

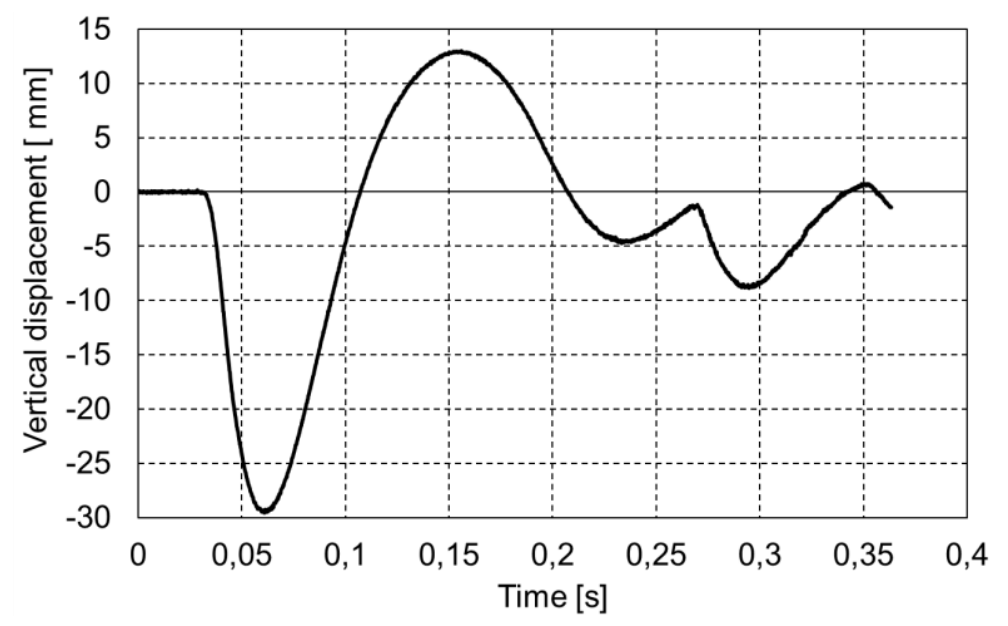

Figure 9. Vertical displacement of the p0 point during drop No. 1.

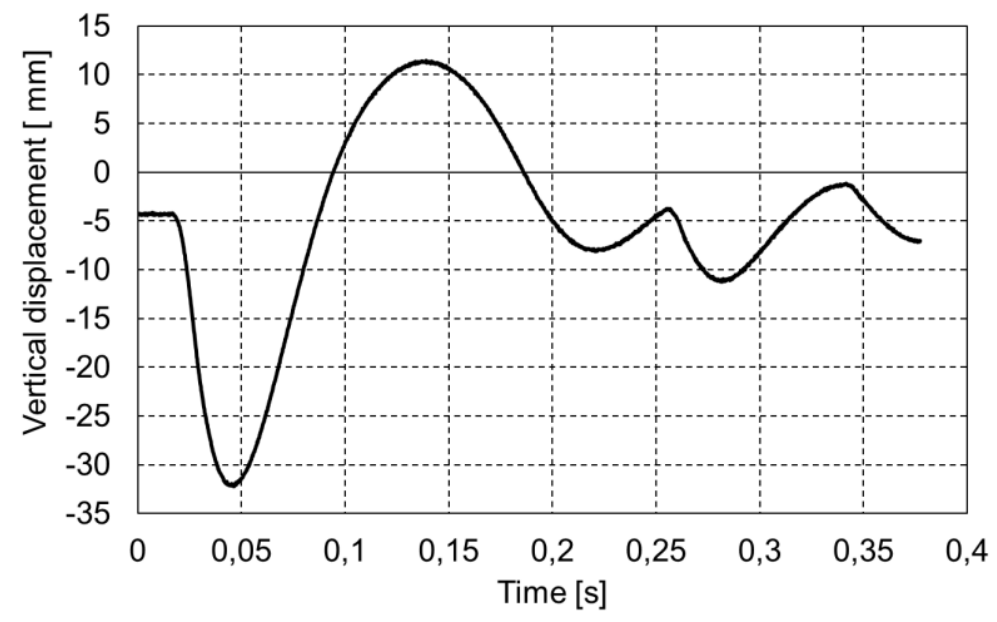

Figure 10. Vertical displacement of the p0 point during drop No. 2. 


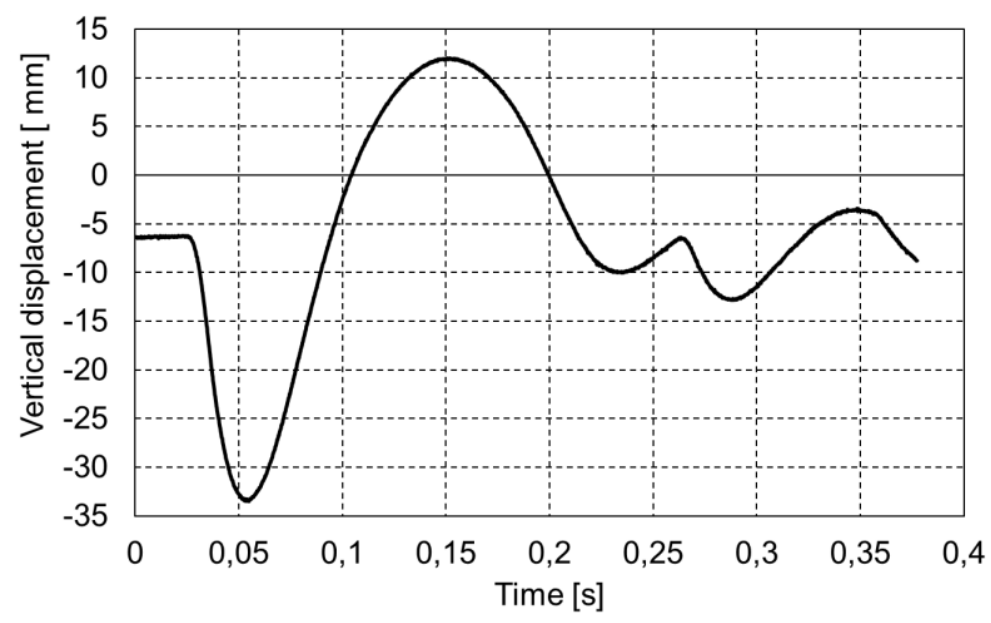

Figure 11. Vertical displacement of the p0 point during drop No. 3.

The values of the measured displacements at individual points during impacts together with the calculated values of the dynamic modulus of deformation Evd are shown in Table 3. The values of Evd indicate a slight strengthening of the test medium after each drop. 
Table 3. The values of measured displacements and dynamic modulus of deformation.

\begin{tabular}{|c|c|c|c|}
\hline & Point p0 & Point p1 & Point p2 \\
\hline \multicolumn{4}{|c|}{ Drop 1} \\
\hline Displacement [mm] & 29,48 & 29,21 & 29,35 \\
\hline $\begin{array}{l}\text { Dynamic modulus of defor- } \\
\text { mation Evd }[\mathrm{kPa}]\end{array}$ & 771,78 & 778,91 & 775,19 \\
\hline \multicolumn{4}{|c|}{ Drop 2} \\
\hline Displacement [mm] & 27,94 & 28,14 & 28,30 \\
\hline $\begin{array}{l}\text { Dynamic modulus of defor- } \\
\text { mation } E_{v d}[\mathrm{kPa}]\end{array}$ & 814,31 & 808,52 & 803,96 \\
\hline \multicolumn{4}{|c|}{ Drop 3} \\
\hline Displacement [mm] & 27,14 & 27,43 & 27,42 \\
\hline $\begin{array}{l}\text { Dynamic modulus of defor- } \\
\text { mation Evd }[\mathrm{kPa}]\end{array}$ & 838,32 & 829,46 & 829,76 \\
\hline
\end{tabular}

\subsection{Minimum values of the weak soil parameters for test shooting}

Taking into account the similar (dynamic) action of the mortar plate and the dynamic load plate, the dynamic modulus of deformation can be adopted as a $E_{v d}$ parameter characterizing the weak soil in terms of test shooting. Using the formula (1), it is possible to estimate the minimum dynamic modulus of deformation of the weak soil, at which it will be possible to safely fire a mortar. For this purpose, it was assumed the Poisson's ratio of peat $v=0,2$, the diameter of the mortar base plate $d_{m}=33 \mathrm{~cm}$ and $\omega=0,79$ as for a circular rigid plate [25]. In addition, a possible displacement of the mortar's base plate should be assumed, which will allow a safe shot to be fired $\Delta s_{\max }=50 \mathrm{~mm}$. The maximum pressure under the base plate of a $60 \mathrm{~mm}$ mortar is $\Delta p_{\max }=1,57 \mathrm{MPa}$. Thus, we get:

$$
E_{v d}^{\min }=\left(1-0,2^{2}\right) \cdot 0,79 \cdot \frac{0,33 \cdot 1,57 \cdot 10^{6}}{0,050}=7,86 \mathrm{MPa}
$$

The weak substrate should therefore be prepared in such a way that the dynamic modulus is larger than $E_{v d}^{\min }$. Due to the fact that the dynamic plate is not adapted to study the deformation modulus of the weak substrate, when determining the modulus $E_{v d,}$ it is necessary to use a high-speed camera. However this procedure is difficult to carry out in the field conditions. A simpler test is to evaluate the static modulus on the basis of a static load test. In the case of the proposed type of weak soil, the relationship between the static and dynamic modulus can be determined. The dependency will apply only to the chosen type of soil. In the presented case, the average primary static modulus is $E=144,6 \mathrm{kPa}$ (see Table 2). The average dynamic modulus after the third drop is $E_{v d}=832,5 \mathrm{kPa}$ (see Table 3). Thus, we get a coefficient of proportionality:

$$
a=\frac{E_{v d}}{E}=5,8
$$

In the case of the tested soil, it can be assumed that the minimum static modulus that must be obtained in order for the substrate to meet the assumed conditions is:

$$
E^{\min }=\frac{E_{v d}^{\min }}{a}=\frac{7,86 \mathrm{MPa}}{5,8}=1,36 \mathrm{MPa}
$$

At this stage, the coefficient $a$ should be determined each time at the initial stage of selection of the soil, because it could be different for various type of organic soil.

In the calculation of the static modulus, the coefficient as for a rigid circular plate is used (see formula (1)). However, it is possible to carry out static test loads using a rigid square-shaped plate. In this case, the shape factor $\omega$ in the formula (1) shall be 0,88 [25].

The determined values of the modules for the model soil are lower than the minimum calculated values, however in the opinion of the authors, verification of the suitability of the proposed substrate in the conditions of firing range is required.

\section{Medium soil}


To characterize the substrate by the dynamic deformation modulus, preliminary field tests combined with shot tests with a $98 \mathrm{~mm}$ mortar in the conditions of the firing range were carried out.

According to the standard [16], the medium soils are sandy, clayey and turf soil. As part of the assessment of the value of the dynamic sandy soil deformation modulus (fine sands - FSa), three tests were made with a lightweight deflectometer. The research was carried out within the shooting position at a depth of $0,5 \mathrm{~m}$, after removing the top layers of sandy soil and humus. The determined average values of soil settlement are in the range of $0,527 \div 0,399 \mathrm{~mm}$, and the dynamic modulus in the range of $42,69 \div 56,39 \mathrm{MPa}$ with an average value of 48,17 MPa.

As part of the assessment of the density of sandy soil, dynamic probing using DPL was performed. The results of test is shown in Figure 12.

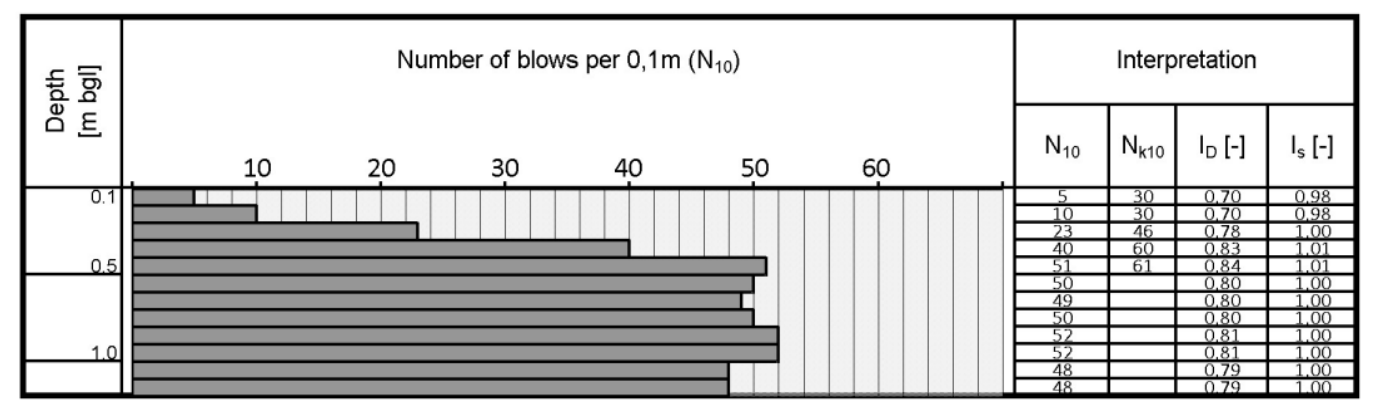

Figure 12. Dynamic probe profile.

The value of the density index $I_{D}$ and the degree of compaction $I_{s}$ are determined respectively from the relationships:

$$
\begin{gathered}
I_{D}=0,429 \log N_{(k) 10}+0,071 \quad[-] \\
I_{S}=\frac{0,818}{0,958-0,174 I_{D}} \quad[-]
\end{gathered}
$$

At the shooting place, four shots were fired. The first two shots were intended to preadjust the plate to the ground. The recoil pulses were 2153,84 and 2828,7 [Ns], respectively. Two next shots were carried out on a maximum pressure propelling charge (for mortar's endurance tests) and the recoil pulse achieved 3704,07 [Ns]. The maximum measured displacements of the base plate during shots No. 3 and 4 were 38,44 and $38,67 \mathrm{~mm}$, respectively. The displacement of the base plate during the third shot is shown in Figure 13.

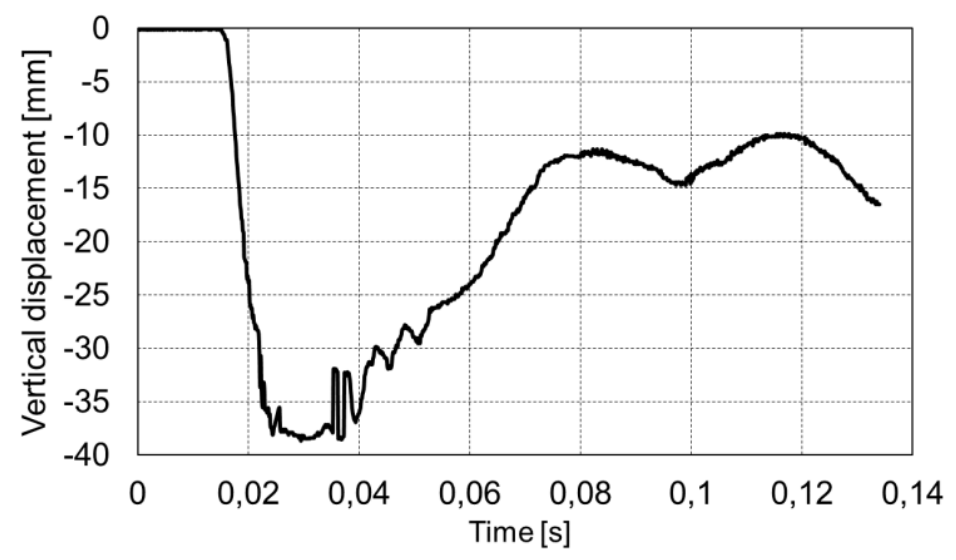

Figure 13. Displacements of the base plate during the third shot. 
Using the results of the maximum displacements of the base plate and assuming in formula (2): $v=0,3$ for sand, $d_{m}=80 \mathrm{~cm}$, the value of the dynamic deformation modulus $E_{v d}$ from the firing pulse load can be determined. The maximum pressure under the base plate of a mortar caliber $98 \mathrm{~mm}$ is $\Delta p_{\max }=1,9 \mathrm{MPa}$, and the average maximum displacement from two mortar shots $\Delta s_{\text {śrmax }}=38,55 \mathrm{~mm}$.

$$
E_{v d}=\left(1-0,3^{2}\right) \cdot 0,79 \cdot \frac{0,8 \cdot 1,9 \cdot 10^{6}}{0,03855}=28,3 \mathrm{MPa}
$$

The obtained value of $28,3 \mathrm{MPa}$ is lower than the value obtained from the dynamic plate test $(48,17 \mathrm{MPa})$. The above can be influenced by a number of factors, the most important seem to be:

- the tube of the mortar and the base plate during the shot are not perpendicular to each other, it may cause slight lateral displacements of the plate,

- dependence of the deformation modulus on the stress value

- the shape factor $\omega$ was adopted as for a rigid circular plate, which can be a certain approximation in the case of a mortar base plate, which is not perfectly rigid and has special ribbing,

- the soil inertia have the higher influence to the speed of deformations during mortar firing than at research test.

\section{Conclusions}

Designing standardized test stands with mortar test sites is a complex issue. In order to ensure the repeatability of the test conditions, the characteristics of the shot process and the cooperation of the mortar with the soil substrate should be taken into account. It is also important in order to confirm that the mortar meets the requirements, including the safety of its operation. The use of standardized soils (with a parametric description of their geotechnical properties) and practical measurement methods will allow for an unambiguous and correct implementation of the mortar certification process. Due to the specificity of shooting tests, the development of stations and research methods that can be used in field conditions. At the moment, there are no such stands or research methods.

The research and results presented in the paper constitute the first approach to the development of a standardized test stands (including soft soils). The requirements for the stands require an innovative approach to the issue of the object's impact on the ground in terms of geotechnical engineering. It is caused both by a much wider range of soil properties (including weak soil, not used in construction), and many times higher loads generated by the mortar. In the next stage, experimental verification of methods and calculations will be carried out by conducting a shooting test.

In the case of medium soils, the sandy subsoil was parameterized in field conditions. The deformation modulus from the lightweight dynamic plate test was determined, as well as the compaction parameters based on DPL probing. The value of the dynamic modulus of deformation was calculated based on the displacement of the mortar base plate as a result of the shot pulse.

The determined values do not coincide, which is due to a number of factors related to the peculiarities of the mortar shot. Further studies are required to determine the influence of the various factors on the results obtained and to determine a sufficiently accurate correlation between the results obtained by means of the presented research methods and shooting tests.

An important aspect of the research is the influence of stress level and dynamics of deformations on the soils properties.

The carried out tests and their development will allow for more accurate modeling of the process of cooperation of the mortar with the substrate during the shot. This is also essential when designing mortars, including ensuring the correct strength of the base plate, ensuring better stability and accuracy of the mortar, and reducing its weight. 
A comparison of the magnitude and dynamics of the loads generated by the mortar and standard measuring devices used in geotechnics shows that the use of a mortar could allow geotechnical investigations in a much wider range of loads.

Author Contributions: Conceptualization, G.B. and K.J.; methodology, M.S.; software, K.J.; validation, T.K., J.L. and G.B.; formal analysis, M.M.; investigation, M.C.; resources, M.S.; data curation, M.S.; writing - original draft preparation, T.K and K.J.; writing - review and editing, M.M.; visualization, M.C.; supervision, G.B. and K.J.; project administration, G.B., K.J. and M.M.; funding acquisition, M.M. All authors have read and agreed to the published version of the manuscript.

Funding: This research received no external funding.

Institutional Review Board Statement: Not applicable.

Informed Consent Statement: Not applicable.

Data Availability Statement: Data sharing is not applicable to this article.

Conflicts of Interest: The authors declare no conflict of interest.

\section{References}

1. R. Kostrow, M. Magier, Z. Pankowski, Artillery of the XXI century [in Polish]; Oficyna Drukarska Jacek Chmielewski, Warsaw, 2006;

2. Magier, M., Ruliński, P., The reliability firing tests of the special ammunition for $98 \mathrm{MM}$ mortar M-98 [in Polish], Journal of Konbin, 2014, 29(1), pp. 81-92, DOI 10.2478/jok-2014-0009;

3. Toivola, J., Moilanen, S., Jussila, H-R.; Force, pressure and strain measurements for traditional heavy launch cycle; Rakenteiden Mekaniikka 44(4):309-329, 2012;

4. Littlefield, A., Sibilla, J.; Simulated Proof Testing of Mortar Baseplates; $26^{\text {th }}$ Army Societe Conference 2008;

5. Wang, F., Yang, G.; Topological Design of a Mortar Base Plate under Impact Loads; Shock and Vibrations Volume 2021; Article ID 8845019;

6. Kosanke, K., Weinman, L.; Typical Mortar Recoil Forces for Spherical Aerial Shell Firings; Fireworks Buisness, No. 242, 2004;

7. Merda, T., Magier, M.; Experimental and numerical analysis of supersonic mortar projectiles; Proceedings - 30th International Symposium on Ballistics, BALLISTICS 2017, 2017, 1, pp. 92-98, DOI 10.12783/ballistics2017/16783;

8. Merda, T., Magier, M.; Selected problems of the construction of $120 \mathrm{~mm}$ high-explosive mortar shells [in Polish]; 2015, Biuletyn PTU WITU, Volume 133 No 1/2015, pp. 21-41;

9. Merda, T., Magier, M.; Comparison analysis of drag coefficients for supersonic mortar projectiles; 2016, Biuletyn PTU WITU, Volume 140 No 4/2016, pp. 21-28;

10. Merda, T., Magier, M.; The Estimation of the Drag Shape for Supersonic Mortar Projectiles; 2017, Machine Dynamics Research 2017, Vol. 41, No 1, 45-52;

11. Merda, T., Magier, M.; Calculations of pitch at flight for mortar projectiles; 2018, Machine Dynamics Research 2018, Vol. 42, No 4, 5-16;

12. Wisniewski A., Pacek D., Zochowski P., Wierzbicki L., Kozlowska J., Zielinska D., Delczyk-Olejniczak B., Struszczyk M.H., Leonowicz M. ,Grabowska G. ,Olszewska K.; Optimization of the material systems with magnetorheological fluids, Proceedings - 28th International Symposium on Ballistics, BALLISTICS 2014 Volume 2, Pages 1602 - 1612, 2014, ISBN 978-160595149-2

13. Wisniewski A., Pacek D., Zochowski P., Wierzbicki L., Kozlowska J., Zielinska D., Delczyk-Olejniczak B., Struszczyk M.H., Leonowicz M. ,Grabowska G. ,Olszewska K., Optimization of the material systems with shear-thickening fluids Proceedings 28th International Symposium on Ballistics, BALLISTICS 2014 Volume 2, Pages 1613 - 1622, 2014, ISBN 978-160595149-2

14. Firing tables to $98 \mathrm{~mm}$ mortar [in Polish];

15. NO-10-A216:2012, Artillery guns - Towed and portable mortars. Requirements and tests [in Polish]

16. PN-EN ISO 14688-1:2018, Geotechnical investigation and testing - Identification and classification of soil - Part 1: Identification and description [in Polish]

17. PN-EN ISO 14688-2:2018, Geotechnical investigation and testing - Identification and classification of soil - Part 2: Principles for a classification [in Polish]

18. Głażewski M., Nowocień E., Piechowicz K.: Earthworks and reclamation works in communication construction [in Polish]. WKE, Warsaw 2011;

19. BN-77/8931-12:1977, Determination of soil compaction index [in Polish];

20. PN-S-02201:1987P, Roads pavements. Classification, definitions and nomenclature. [in Polish]. PKN, Warsaw 1987.

21. Łupieżowiec, Marian Large-size test loads as a method of recognizing the bearing capacity and stiffness of the ground substrate [in Polish]. Technical Journal. Environment R. 104, Issue 12, 1-Ś, 2007 Kraków;

22. Kudla W., Floss R., Trautmann C.: Dynamischer Plattendruckversuch - Schnellprüfverfahren für die Qualitätssicherung von unbound Schichten. Straße und Autobahn [in German], Vol. 2, Bonn 1991, 66-71;

23. Weingart W.: Problems of dynamic load capacity testing with drop devices [in German]. Die Strasse, No. 11, Berlin 1981, 369_ 373 ; 
24. Gosk W.: Identification of the ground stiffness modulus using oscillators [in Polish]. Scientific Notebooks of Bialystok University of Technology - Construction, Z. 32, Bialystok University of Technology, Bialystok 2008, 45-62;

25. Wiłun Z., Zarys geotechniki [in Polish], 1987, WKŁ, Warsaw. 